\title{
Memory Consolidation and Reconsolidation in the Rat Pup Require Protein Synthesis
}

\author{
Nadège Gruest, Paulette Richer, and Bernard Hars \\ Laboratoire de Neurobiologie de l'Apprentissage, de la Mémoire, et de la Communication, Centre National de la Recherche Scientifique, Unité Mixte de \\ Recherche 8620, Université Paris-sud, 91405 Orsay Cedex, France
}

Little is known about the ontogenesis of memory, whether it appears with its full characteristics or whether they emerge progressively with development. In the adult, basic characteristics of memory processing are consolidation of memory after acquisition and reconsolidation after retrieval. Here, using a conditioned aversion paradigm and postlearning or postreactivation injection of a protein synthesis inhibitor, we show that memory acquired by rat pups as early as postnatal day 3 requires time-dependent protein synthesis after both learning and reactivation. These results present the first evidence suggesting that consolidation and reconsolidation are original properties of memory function.

Key words: memory; consolidation; reconsolidation; protein synthesis; conditioned aversion; rat pup

\section{Introduction}

At birth, the newborn has to cope with dramatic environmental changes and must acquire many adaptive responses. It needs to encode and retain new information to update its competencies and select relevant behavioral adaptations to survive in its new world. The ability to acquire new information and form memories has been demonstrated in mammals at birth; however, the properties and dynamics of this early memory have not yet been characterized. One of the main characteristics of adult memory is a time-dependent change, such that new memories are initially in a labile state that marks the transition from short-term to longterm memory, during which memory is consolidated (McGaugh, 1966). In the adult, there is a consensus that the consolidation of new memories require de novo synthesis of proteins (Davis and Squire, 1984). Recent research has also revived the idea that when retrieved, previously consolidated memories become temporarily labile again and may require a further stabilization process referred to as reconsolidation, to remain in long-term memory and be available for later recall (Lewis, 1979; Sara, 2000; Nader, 2003). In terms of the underlying mechanisms, several studies have provided evidence that both processes of consolidation and reconsolidation require protein synthesis (Judge and Quartermain, 1982; Nader et al., 2000; Debiec et al., 2002; Kida et al., 2002). It is not known, however, whether these time and protein synthesis-dependent characteristics of memory consolidation and reconsolidation are already present at birth or whether they emerge progressively with brain maturation and the develop-

Received July 22, 2004; revised 0ct. 11, 2004; accepted 0ct. 11, 2004.

We express gratitude to Sabrina Davis and Serge Laroche for critical comments on this manuscript, to Cyrille Vaillend for help with the statistical analysis, and to Pascale Veyrac, Nathalie Samson, and Gérard Dutrieux for their technical assistance.

Correspondence should be addressed to Bernard Hars at the above address. E-mail: bernard.hars@ ibaic.u-psud.fr.

DOI:10.1523/JNEUROSCI.2984-04.2004

Copyright $\odot 2004$ Society for Neuroscience $\quad$ 0270-6474/04/2410488-05\$15.00/0 ment of cognitive skills. To address this issue, we used a conditioned taste aversion (CTA) paradigm in 3-d-old rat pups (P3) and investigated the effect of injecting the protein synthesis inhibitor anisomycin (AN) at varying delays after learning and after reactivation of an already established aversion memory. CTA (Garcia et al., 1966) induces robust memory for particular flavors when they are associated with poison after ingestion. Animals learn to avoid these foods as a vital protective reaction against repeated intake of dangerous foods, and this memory ability is already present in early life (Kehoe and Blass, 1986; Hoffmann et al., 1987; Gruest et al., 2004).

\section{Materials and Methods}

Subjects. Male and female Wistar rat pups (originating from parent Wistar rats; Iffa Credo, L'Arbresle, France) were used as subjects. On the day of birth (P0), litters were culled to eight pups. The mothers and their litters were housed in standard maternity cages and maintained in a temperature-controlled colony room on a $12 \mathrm{hr}$ light/dark cycle. All experimental procedures were performed in conformity with national (JO 887-848) and European (86/609/EEC) legislation.

Training procedure. We used a CTA paradigm in 3-d-old pups in which the conditioned stimulus (CS) (peach-aromatized milk; Régilait, SaintMartin de Belle-Roche, France) was given via a surrogate nipple for 10 min and paired $30 \mathrm{~min}$ later with an injection of $0.15 \mathrm{M} \mathrm{LiCl}$ (2\% body weight, i.p.; Sigma, St. Quentin Fallavier, France) that caused an immediate malaise (unconditioned stimulus, US). Control pups were injected with $0.9 \% \mathrm{NaCl}$ ( $2 \%$ body weight i.p.). Pups were isolated from the dam about $11 \mathrm{hr}$ before training on $\mathrm{P} 3$ and were exposed to an artificial nipple apparatus (Petrov et al., 1997). The surrogate nipple was a latex teat (35 $\mathrm{mm}$ long, $3.5 \mathrm{~mm}$ in diameter; Catac Products, Bedford, UK) that was gently presented to the pup, and the contact with the perioral region elicited an oral grasp response followed by active attachment to the teat.

We chose this protocol because Petrov et al. (1997) in a well documented set of experiments showed that the artificial nipple technique was a highly effective tool for investigating the attractive or aversive value of a particular fluid, thereby allowing a robust means for testing conditioning in the newborn rat (Cheslock et al., 2000; Nizhnikov et al., 2002a). We 
used this technique for the first time in a CTA protocol, the advantage of which was to avoid surgery and anesthesia required for the intraoral cannulation technique often used previously in CTA in newborn (Kehoe and Blass, 1986; Hoffmann et al., 1987), therefore avoiding treatments that could interfere with consolidation and reconsolidation.

Anisomycin administration. Anisomycin (Sigma), dissolved in $0.9 \%$ saline and adjusted to $\mathrm{pH} 7.2$ with $1 \mathrm{~N} \mathrm{HCl}$, was injected ( $42 \mathrm{mg} / \mathrm{kg}$, i.p.). Control pups were injected with $0.9 \%$ saline. To ensure independence of the data, only one pup from a litter was assigned to a given condition and treatment group.

Reactivation treatment. To equalize reactivation between pups, intraoral contact with the surrogate nipple filled with aromatized milk, the nipple was imposed on the pups by putting it into their mouth for a $5 \mathrm{~min}$ period on P5. Before cueing, all pups were isolated from the dams as for conditioning.

Testing. On day 6 (consolidation) or day 7 (reconsolidation), the CS was presented in the morning for a 10 min session after $\sim 11 \mathrm{hr}$ of isolation from the dam. During testing as with conditioning, the attractiveness of aromatized milk was assessed by the time pups spent attached to the nipple and the weight gain of pups during the suckling session. The two measures were generally well correlated, and we used the time of attachment as it appeared a more discriminative measure and showed better expression of the active response of the pups. This higher level of sensitivity of attachment has been shown before by Nizhnikov et al. (2002b). The total time of attachment to the nipple was measured on-line by two experimenters who were blind to the pretreatment of the pups.

Data analysis. A taste aversion index was calculated as the relative time spent attached to the nipple (time during testing/total time during conditioning and testing) $\times 100$. This was chosen to limit the potential interindividual variability in the oral grasping response and to take into account the conditioning session. Thus, the more the pups refuse the flavored milk nipple (aversion), the lower the score is. ANOVA and $t$ tests were used for statistical analysis. Data from both male and female pups were combined because ANOVA confirmed that there was no sex difference in any of the measures reported here.

\section{Results}

\section{Anisomycin impairs formation of an early memory}

Experiment 1 was designed to test whether pups could learn aversion in the CTA protocol and whether this memory underwent a protein synthesis-dependent formation at the early age of $\mathrm{P} 3$. To control the establishment of conditioned aversion in the CS-US condition, we used two other conditions, either the CS was presented alone or the US preceded the CS by $10 \mathrm{hr}$. The effect of protein synthesis inhibition was tested in these three conditions by $\mathrm{AN}$ injections given $15 \mathrm{~min}$ after the CS.

The results are presented in Figure 1. A two-way ANOVA revealed a significant protocol $X$ anisomycin treatment interaction $\left(F_{(2,58)}=5.01 ; p=0.009\right)$ with a main effect of protocol $(p<0.0001)$ and of treatment $(p=0.002)$, indicating a differential effect of AN on paired and nonpaired groups. In the three groups without AN, the pups that were exposed to the novel taste paired with the malaise (CS-US), attached to the nipple significantly less than pups in the two control groups (CS alone or backward US-CS). For these pups there was a main effect of group $\left(F_{(2,30)}=18.15 ; p<0.0001\right)$ with significant differences between paired and control pups ( $p<0.001$ in each case). In contrast, when AN was injected 15 min after the CS in the CS-US group, a complete abolition of the aversion to the aromatized milk was observed, these pups were not different from those of the two control AN-treated groups (CS-AN or US-CS-AN) $\left(F_{(2,28)}=1.3 ; p>0.05\right)$. AN, however, had no effect on performance in the CS alone or US-CS groups, there was no significant difference between these groups regardless of whether they were injected with $\mathrm{AN}$ or not $(p>0.05$ in each case), suggesting that $\mathrm{AN}$ had no effect per se on suckling behavior measured during

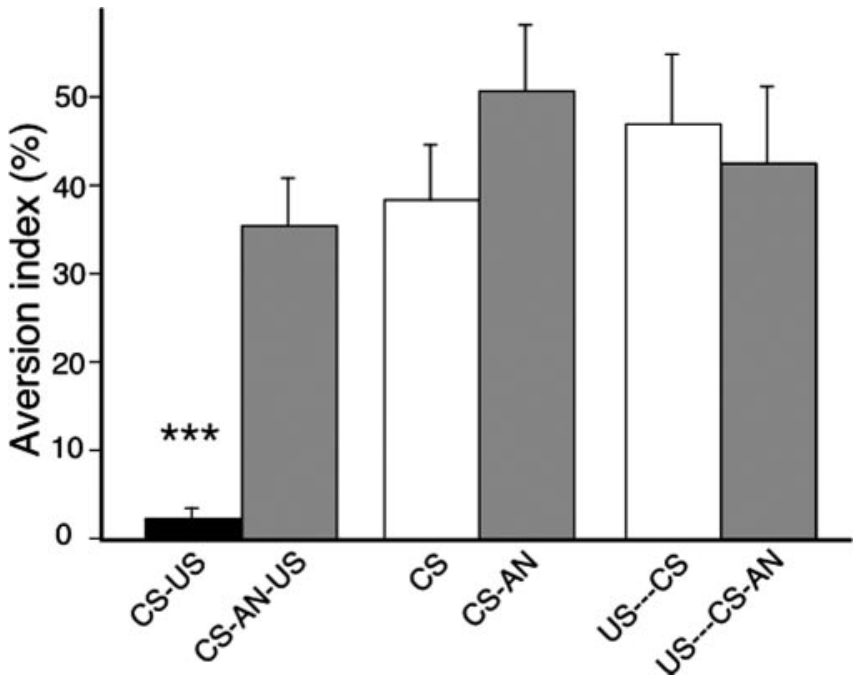

Figure 1. Effect of anisomycin given during training. Percentage of time attachment to the surrogate nipple on the test for the six groups of rat pups. The lower the index, the more the aversion. Pups were trained on P3 and tested on P6. Pups of group CS-US $(n=11)$ received aromatized milk paired with $\mathrm{LiCl}$, and those of CS-AN-US $(n=10)$ received anisomycin between CS and US. Two control conditions were used: aromatized milk alone (CS; $n=10)$ and $\mathrm{LiCl}$ preceding milk from $10 \mathrm{hr}$ (US-CS; $n=12$ ). The effect of AN was tested in these two conditions (CS-AN; $n=12$ ) and (US-CS-AN; $n=9$ ). Error bars indicate SE. ${ }^{* *} p<0.001$. Note that anisomycin abolishes the conditioned aversion.

testing. These results show that pups at P3 can learn and form a long-term conditioned aversion memory and that protein synthesis is required for formation of this early memory.

\section{Anisomycin disrupts consolidation of an early memory}

Experiment 2 was designed to determine the temporal gradient of efficacy of AN on aversion memory by varying the interval between conditioning at $\mathrm{P} 3$ and $\mathrm{AN}$ injections. Therefore, $\mathrm{AN}$ was delivered at different time points after the CS-US presentation either immediately or at $15 \mathrm{~min}, 1 \mathrm{hr}, 6 \mathrm{hr}$, and $24 \mathrm{hr}$. A new unpaired control condition was used, the CS preceding the US from $10 \mathrm{hr}$, and the AN effect was also tested in this condition.

The results are presented in Figure 2. First, comparison of anisomycin treatment immediately after pairing versus unpairing showed a significant protocol $\times$ treatment interaction $\left(F_{(1,44)}\right.$ $=5.007 ; p=0.03)$. The CS-US group showed a marked aversion to the aromatized milk when compared with CS-US unpaired groups and the CS-US + AN0 paired group $(p<0.01$ in each case); thus replicating the results of experiment 1 and reinforcing the associative nature of the aversion. Second, when anisomycin was injected at increasing delays after conditioning, a temporally graded effect was observed (one-way ANOVA, delays 0-24 hr; $\left.F_{(4,43)}=3.41 ; p=0.01\right)$. AN injected immediately or $15 \mathrm{~min}$ after conditioning resulted in a profound impairment of aversion memory when compared with CS-US nontreated pups $(p<0.01$ in each case), whereas the impairment of memory was reduced at $1 \mathrm{hr}$ and completely absent at 6 and $24 \mathrm{hr}$, delays at which pups showed a level of aversion similar to that of the CS-US nontreated group ( $p>0.05$ in each case). These later results rule out a possible anterograde effect and argue for retrograde amnesic effect of $A N$ injection. In all, the results of experiment 2 show that the aversion memory in pups becomes increasingly stable over time until it becomes immune to the disruptive effect of protein synthesis inhibition and define a temporal gradient of protein synthesis-dependent consolidation of this type of memory in pups. 


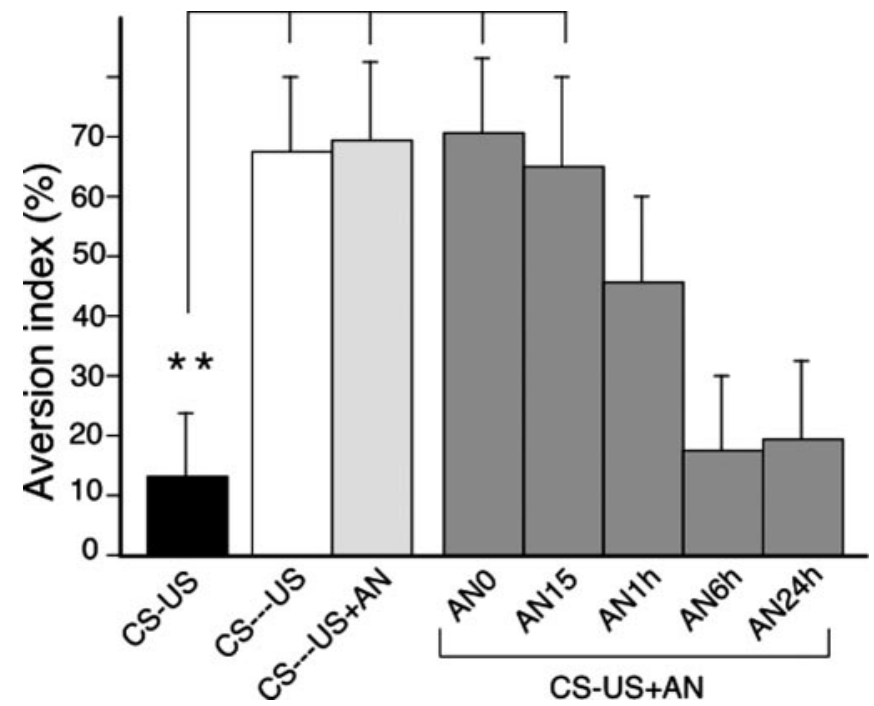

Figure 2. Effect of anisomicin given after training. Pups of group CS-US $(n=10)$ received aromatized milk paired with LiCl without anisomycin treatment. In the other paired groups, pups received anisomycin at different delays, either immediately (ANO; $n=11$ ) or $15 \mathrm{~min}$ $($ AN15; $n=9), 1 \mathrm{hr}($ AN1h; $n=9), 6 \mathrm{hr}$ (AN6h; $n=9)$, or $24 \mathrm{hr}$ (AN24h; $n=10$ ) after training. Two groups were submitted to an unpaired condition with a long delay between aromatized milk presentation and LiCl either without anisomycin (CS-US; $n=14$ ) or followed by anisomycin (CS-US $+\mathrm{AN} ; n=13)$. Error bars indicate $S E .{ }^{* *} p<0.01$. Note that anisomycin abolishes the conditioned aversion at 0 and 15 min delay; at 6 and $24 \mathrm{hr}$ it has no more effect.

\section{Anisomycin disrupts reconsolidation of an early memory}

To examine whether an established early aversion memory becomes labile when retrieved and requires a phase of protein synthesis to remain available for later recall, a brief reactivation episode was interposed on P5 between training and test. AN was injected at varying delays after reactivation: either immediately or at $15 \mathrm{~min}, 30 \mathrm{~min}, 1 \mathrm{hr}, 6 \mathrm{hr}$, and $24 \mathrm{hr}$. Two additional controls were used: an unpaired (CS preceding US from $10 \mathrm{hr}$ ) but reactivated group, and a paired CS-US group in which pups received $\mathrm{AN}$ on P5, but were not reactivated. Initial training on P3 and testing on P7 were performed as previously described.

As seen in Figure 3, pups in the CS-US conditioned group that was reactivated on $\mathrm{P} 5$ but did not receive AN showed a much greater aversion score compared with pups of the unpaired group $(p<0.0001)$, suggesting that the brief reactivation session did not cause extinction of the memory. As observed in experiment 2 the delay determined the efficiency of AN treatment (one-way ANOVA, delays $\left.0-24 \mathrm{hr} ; F_{(5,66)}=92.44 ; p<0.0001\right)$. When AN was injected 0,15 , or 30 min after reactivation, a marked suppression of aversion was observed in these groups when compared with the reactivated CS-US group without AN $(p<0.0001$ in all cases). This impairment in aversion memory progressively decayed as the delay between reactivation and AN injection increased: aversion was still reduced in the group with the delay of $1 \mathrm{hr}$ when compared with the reactivated CS-US group without AN $(p<0.0001)$, but the level of aversion was significantly greater than in the groups with $\mathrm{AN}$ at shorter delays $(p<$ 0.0001). At 6 and $24 \mathrm{hr}$ after reactivation, AN no longer had any deleterious effect on the aversion memory, with both groups showing a similar level of aversion to that of the conditioned nontreated pups. In addition, the group of pups that received AN on P5 but without cueing showed normal aversion to the milk, demonstrating that reactivation of the memory was necessary to render the memory labile and susceptible to disruption again. The results therefore suggest that the early memory trace under-

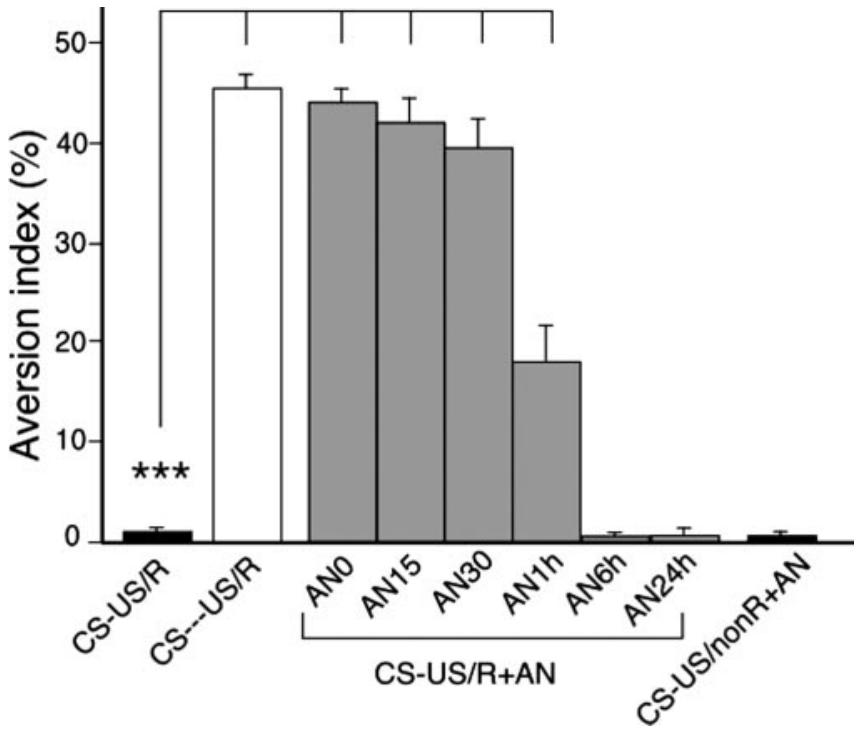

Figure 3. Effect of anisomycin given after reactivation. After training on P3, pups were cued on $\mathrm{P} 5$ by a brief presentation of aromatized milk followed by anisomycin treatment at different delays; test was on P7. One group was paired and reactivated without anisomycin (CS-US/R; $n=11$ ), and the other ones received anisomycin either immediately (AN0; $n=12$ ) or $15 \mathrm{~min}$ $($ AN15; $n=12), 30 \mathrm{~min}(\operatorname{AN} 30 ; n=12), 1 \mathrm{hr}(\operatorname{AN} 1 \mathrm{~h} ; n=12), 6 \mathrm{hr}($ AN6h; $n=12)$, or $24 \mathrm{hr}$ (AN24h; $n=12$ ) after cueing. A control group was unpaired but reactivated (CS-US/R; $n=$ 12), and another one was paired and received anisomycin on $P 5$, but was not reactivated (CS-US/nonR + AN; $n=12$ ). Error bars indicate SE. ${ }^{* *} p<0.0001$. Note that anisomycin is able to impair conditioned aversion $48 \mathrm{hr}$ after training but only if preceded by reactivation, and its effect is time-dependent.

goes reconsolidation after reactivation and follows a similar protein synthesis-dependent time course as consolidation.

\section{Discussion}

These results indicate that memory formed at a very early age is dependent on the synthesis of new proteins either after acquisition or reactivation. They are in keeping with a growing body of evidence in the adult that shows a time-dependent sensitivity of memory whenever it is in active state (Lewis, 1979; Sara, 2000; Riccio et al., 2002; Nader, 2003), indicating the memory trace undergoes a form of processing in consolidation and reconsolidation. We show here these processes are already involved in early memories.

Our data address several important questions. Two that are currently still under debate concern the content and structure of memories. The first question concerning consolidation is whether the newly consolidated memory is an independent entity or whether it becomes integrated and updates previously existing memories. This question has been mainly directed toward the process of reconsolidation (Przybyslawski and Sara, 1997; Nader, 2003), in which competition between the reactivated trace and the established trace appears evident, but it can also be directed toward consolidation, in which the new trace may compete with previously unidentified stored information. Although the pups were only at a P3 stage, we cannot assume the memory bank to be completely void at this time because the capacity for learning and memory exists before this period (Stickrod et al., 1982; Abate et al., 2001; Gruest et al., 2004). However, the memory store may still have only a small content that may suggest consolidation at the beginning of life could be primarily implicated in the integration of new information into a genetically programmed repertoire, as implied by Seligman (1970). The second question concerns the structure of memory. The fact that re-exposure of 
animals to a fragment of the original learned association is capable of reactivating the memory trace is consistent with the view proposed by Spear (1973) that memory is multidimensional. Many studies have shown that different types of reminder can induce retrieval of a memory in various conditions. For example we have previously shown that contextual cues are able to facilitate memory of forgotten maze habits (Deweer et al., 1980) and that a reminder given during sleep may reinduce malleability in memory either during paradoxical sleep or slow wave sleep (Hars et al., 1985; Hars and Hennevin, 1987). Taken together, these data reinforce the notion that activation of an attribute may induce arousal and retrieval of the target memory (Spear, 1973). These data, however, have been demonstrated in the adult animal; the present results show this ability is already present at the beginning of life despite the very rudimentary cognitive capacities at that stage.

The multidimensional nature of the memory trace raises the question as to what is actually learned by the pups at the time of training. They are submitted to a new taste (the CS) as classically used in a CTA paradigm, but other stimuli are also available during CS presentation, such as contextual cues (either external or internal), including the nipple itself and the odor of aromatized milk. Therefore the new taste is not the only cue involved in the aversion, and the gustatory aspect of learning may be only one component of the aversion acquired and tested in our experimental conditions.

In the adult there is consensus that memory formation of CTA is blocked by protein synthesis inhibitors. In most studies the inhibitors have been delivered before the US, thus precluding the possibility of testing a temporal gradient of efficiency after training (Rosenblum et al., 1993; Houpt and Berlin, 1999). However, using these conditions Houpt and Berlin (1999) did show that the inhibitor disrupts long-term CTA memory ( 6 and $48 \mathrm{hr}$ ) but not short-term memory $(1 \mathrm{hr})$, and the authors propose that protein synthesis before $4.5 \mathrm{hr}$ is required to successfully consolidate CTA. This time window is compatible with our results in the pup. When a CTA memory is retrieved Eisenberg et al. (2003) show that AN may have different effects, depending on the strength of the original trace: AN blocks extinction of a weak memory trace thereby protecting the original one, but disrupts reconsolidation of a strong trace, thereby impairing the original memory. Here we show that reactivation of the early memory leads to impairment as seen in the adult when the trace is strong enough.

The similarity in the temporal gradients after acquisition and reactivation observed in our experiments suggests that consolidation and reconsolidation share similar mechanisms at the cellular level. It is arguable however, whether different networks occurring within a similar temporal window may be implicated. Because we used systemic administration of AN, we are not in a position to address the issue of which neural systems and brain structures are involved in the formation, consolidation, and reconsolidation of early conditioned aversion memory. Importantly it has been shown that the cerebral cortex is not fully developed in the first postnatal week (Bayer et al., 1993) and the gustatory cortex, which is clearly implicated in formation of CTA memories in adults (Rosenblum et al., 1993; Berman and Dudai, 2001), may be compensated for by deeper structures of the brain in early life. This suggests that the neuronal circuitry involved in the formation of CTA memories may evolve during development.

Several studies in the adult have shown that both consolidation and reconsolidation of memories require the synthesis of new proteins (Judge and Quartermain, 1982; Nader et al., 2000; Debiec et al., 2002; Milekic and Alberini, 2002). In general, these studies suggest that a cascade of molecular and cellular events initiated by an experience results in durable form of synaptic modification (Geinisman et al., 2001; Silva, 2003). However, questions relating to similarity and difference between the mechanisms required in consolidation and reconsolidation are only beginning to be addressed, for example the specificity of individual proteins and neuronal circuitry (Abel and Lattal, 2001; Debiec et al., 2002; Kida et al., 2002; Bozon et al., 2003; Bahar et al., 2004; Lee et al., 2004). The behavioral model described here will be helpful in future work to dissect out the neural systems and the molecular and cellular mechanisms recruited during consolidation and reconsolidation of a primitive memory.

In summary, to our knowledge the present results are the first demonstration of very early memory consolidation and reconsolidation in the mammal. They show that the dynamics of these processes are present at the beginning of ontogenesis and are comparable with those shown in the adult in different species and different types of memory. They suggest that consolidation and reconsolidation are not emergent but innate properties of memory function.

\section{References}

Abate P, Spear NE, Molina JC (2001) Fetal and infantile alcohol-mediated associative learning in the rat. Alcohol Clin Exp Res 25:989-998.

Abel T, Lattal KM (2001) Molecular mechanisms of memory acquisition, consolidation and retrieval. Curr Opin Neurobiol 11:180-187.

Bahar A, Dorfman N, Dudai Y (2004) Amygdalar circuits required for either consolidation or extinction of taste aversion memory are not required for reconsolidation. Eur J Neurosci 19:1115-1118.

Bayer SA, Altman J, Russo RJ, Zhang X (1993) Times tables of neurogenesis in the human brain based on experimentally determined patterns in the rat. Neurotoxicology 14:83-144.

Berman DE, Dudai Y (2001) Memory extinction, learning anew, and learning the new: dissociations in the molecular machinery of learning in cortex. Science 291:2417-2419.

Bozon B, Davis S, Laroche S (2003) A requirement for the immediate early gene zif268 in reconsolidation of recognition memory after retrieval. Neuron 40:695-701.

Cheslock SJ, Varlinskaya EI, Petrov ES, Spear NE (2000) Rapid and robust olfactory conditioning with milk before suckling experience: promotion of nipple attachment in the newborn rat. Behav Neurosci 114:484-495.

Davis HP, Squire LR (1984) Protein synthesis and memory: a review. Psychol Bull 96:518-559.

Debiec J, LeDoux JE, Nader K (2002) Cellular and systems reconsolidation in the hippocampus. Neuron 36:527-538.

Deweer B, Sara SJ, Hars B (1980) Contextual cues and memory retrieval in rats: Alleviation of forgetting by a pretest exposure to background stimuli. Anim Learn Behav 8:265-272.

Eisenberg M, Kobilo T, Berman DE, Dudai Y (2003) Stability of retrieved memory: inverse correlation with trace dominance. Science 301:1102-1104.

Garcia J, Ervin FR, Koelling RA (1966) Learning with prolonged delay of reinforcement. Psychon Sci 5:121-122.

Geinisman Y, Berry RW, Disterhoft JF, Power JM, Van der Zee EA (2001) Associative learning elicits the formation of multiple-synapse boutons. J Neurosci 21:5568-5573.

Gruest N, Richer P, Hars B (2004) Emergence of long-term memory for conditioned aversion in the rat fetus. Dev Psychobiol 44:189-198.

Hars B, Hennevin E (1987) Impairment of learning by cueing during postlearning slow-wave sleep in rats. Neurosci Lett 79:290-294.

Hars B, Hennevin E, Pasques P (1985) Improvement of learning by cueing during postlearning paradoxical sleep. Behav Brain Res 18:241-250.

Hoffmann H, Molina JC, Kucharski D, Spear NE (1987) Further examination of ontogenetic limitations on conditioned taste aversion. Dev Psychobiol 20:455-463.

Houpt TA, Berlin R (1999) Rapid, labile, and protein synthesis-independent short-term memory in conditioned taste aversion. Learn Mem 6:37-46.

Judge ME, Quartermain D (1982) Characteristics of retrograde amnesia following reactivation of memory in mice. Physiol Behav 28:585-590.

Kehoe P, Blass EM (1986) Conditioned aversions and their memories in 
5-day-old rats during suckling. J Exp Psychol Anim Behav Process 12:40-47.

Kida S, Josselyn SA, de Ortiz SP, Kogan JH, Chevere I, Masushige S, Silva AJ (2002) CREB required for the stability of new and reactivated fear memories. Nat Neurosci 5:348-355.

Lee JL, Everitt BJ, Thomas KL (2004) Independent cellular processes for hippocampal memory consolidation and reconsolidation. Science 304:839-843.

Lewis DJ (1979) Psychobiology of active and inactive memory. Psychol Bull 86:1054-1083.

McGaugh JL (1966) Time dependent processes in memory storage. Science 153:1351-1358.

Milekic MH, Alberini CM (2002) Temporally graded requirement for protein synthesis following memory reactivation. Neuron 36:521-525.

Nader K (2003) Memory traces unbound. Trends Neurosci 26:65-72.

Nader K, Schafe GE, Le Doux JE (2000) Fear memories require protein synthesis in the amygdala for reconsolidation after retrieval. Nature 406:722-726.

Nizhnikov ME, Petrov ES, Spear NE (2002a) Olfactory aversive conditioning in the newborn (3-hr-old) rat impairs later suckling for water and milk. J Exp Psychol Anim Behav Process 28:277-283.
Nizhnikov ME, Petrov ES, Varlinskaya EI, Spear NE (2002b) Newborn rats' first suckling experience: taste differentiation and suckling plasticity. Physiol Behav 76:181-198.

Petrov ES, Varlinskaya EI, Smotherman WP (1997) The newborn rat ingests fluids through a surrogate nipple: a new technique for the study of early suckling behavior. Physiol Behav 62:1155-1158.

Przybyslawski J, Sara SJ (1997) Reconsolidation of memory after its reactivation. Behav Brain Res 84:241-246.

Riccio DC, Moody EW, Millin PM (2002) Reconsolidation reconsidered. Integr Physiol Behav Sci 37:245-253.

Rosenblum K, Meiri N, Dudai Y (1993) Taste memory: the role of protein synthesis in gustatory cortex. Behav Neural Biol 59:49-56.

Sara SJ (2000) Retrieval and reconsolidation: toward a neurobiology of remembering. Learn Mem 7:73-84.

Seligman M (1970) On the generality of the laws of learning. Psychol Rev 77:406-418.

Silva AJ (2003) Molecular and cellular cognitive studies of the role of synaptic plasticity in memory. J Neurobiol 54:224-237.

Spear NE (1973) Retrieval of memory in animals. Psychol Rev 80:163-194. Stickrod G, Kimble DP, Smotherman WP (1982) In utero taste/odor aversion conditioning in the rat. Physiol Behav 28:5-7. 